\title{
Charge ordering in extended Hubbard models: Variational cluster approach
}

\author{
M. Aichhorn, ${ }^{1}$ H. G. Evertz, ${ }^{1}$ W. von der Linden, ${ }^{1}$ and M. Potthoff ${ }^{2}$ \\ ${ }^{1}$ Institut für Theoretische Physik, Technische Universität Graz, Petersgasse 16, A-8010 Graz, Austria \\ ${ }^{2}$ Institut für Theoretische Physik und Astrophysik, \\ Universität Würzburg, Am Hubland, D-97074 Würzburg, Germany
}

\begin{abstract}
We present a generalization of the recently proposed variational cluster perturbation theory to extended Hubbard models at half filling with repulsive nearest neighbor interaction. The method takes into account short-range correlations correctly by the exact diagonalisation of clusters of finite size, whereas long-range order beyond the size of the clusters is treated on a mean-field level. For one dimension, we show that quantum Monte Carlo and density-matrix renormalization-group results can be reproduced with very good accuracy. Moreover we apply the method to the two-dimensional extended Hubbard model on a square lattice. In contrast to the one-dimensional case, a first order phase transition between spin density wave phase and charge density wave phase is found as function of the nearest-neighbor interaction at onsite interactions $U \geq 3 t$. The single-particle spectral function is calculated for both the one-dimensional and the two-dimensional system.
\end{abstract}

PACS numbers: $71.10 .-\mathrm{w}, 71.27 .+\mathrm{a}, 71.30 .+\mathrm{h}, 75.10 .-\mathrm{b}$

\section{INTRODUCTION}

In recent years an increasing number of theoretical and experimental studies in condensed matter physics have focused on the description and understanding of quasi one and two dimensional strongly correlated electronic systems. Several fascinating properties of these materials are due to the competition between different phases with long-range order. High-temperature superconductivity in cuprates is one of the most famous examples which is not yet understood in a satisfactory way. Realistic models that are used in this context consist of a kinetic part which accounts for the electron motion and an interaction part which is of the same order of magnitude. The simplest model that can be constructed under these assumptions is the tight binding Hubbard model. It consists of a kinetic energy part, where the electrons can only hop between nearest neighbor sites and the Coulomb interaction $U$ which acts only locally on each site. Although this model was used with great success for the description of a wide class of materials, there are interesting physical questions which require an extension. The inclusion of the nearest-neighbor Coulomb interaction, for example, is necessary for the study of inhomogeneous phases, such as the charge-density wave (CDW). This leads to the so called extended Hubbard model (EHM).

But knowing the appropriate model for the description of a material is only the first step on the way to understanding the physics. Already for the simple Hubbard model without non-local Coulomb interaction, an exact calculation of static and dynamic properties is possible in very special cases only and one has to be content with approximate methods in general. For the interesting case where the Coulomb interaction $U$ is of the same order of magnitude as the bandwith $W$, the conventional perturbative approach must fail. This is expected for weak-coupling perturbation theory but also for the complementary approach with exact treatment of the interaction part and perturbative treatment of the kinetic energy: $1,2,3$

Numerical methods are more promising, such as quantum Monte Carlo (QMC), 4 exact diagonalisation (ED), and density-matrix renormalization group (DMRG) $\underline{\underline{5}}$ They are able to give essentially exact results - at least for limited system sizes or (DMRG) for the one dimensional case. Another non-perturbative approach is the mean-field method and, in the context of the Hubbard model, the dynamical mean field theory (DMFT), 6 in particular. While the DMFT directly works in the thermodynamic limit of infinite system size, it must be regarded as a strong approximation since spatial correlations are neglected altogether. Cluster generalizations of the DMFT include at least short-range correlations via the exact treatment of a small cluster instead of considering a single impurity only. Both, a reciprocal-space (dynamical cluster approximation, $\mathrm{DCA}^{7}$ ) and a real-space construction (cellular dynamical mean field theory, C$\mathrm{DMFT}^{8,9,10}$ ) have been suggested.

Essentially the same idea is followed with the cluster perturbation theory (CPT),11.12.13 which is a cluster extension of the strong-coupling expansion for the Hubbard model: The lattice is divided into small clusters which are solved exactly while the hopping between adjacent clusters is treated perturbatively. The lowest order of the strong-coupling expansion in the inter-cluster hopping yields the CPT. Short range correlations on the scale of the cluster are taken into account exactly, for instance by the Lanczos technique at zero temperature, while correlations on a scale larger than the cluster size are neglected. The CPT is a systematic approach with respect to the cluster size, i.e. the method becomes exact in the limit $N_{c} \rightarrow \infty$, where $N_{c}$ is the number of sites within a cluster. It allows for the calculation of the single-electron Green's function at arbitrary values of the wave vector $\mathbf{k}$. This is a considerable improvement compared to standard Lanczos calculations for small clusters, where only a few $\mathbf{k}$ points are available. The CPT has been successfully used to describe spectral properties of the high- $T_{C}$ 
materials,,$\frac{14,15,16}{17}$ and has already been extended to finite temperatures 17

Recently a new method has been proposed which exploits a general variational principle for the self-energy of a system of interacting fermions. This self-energyfunctional approach (SFA) ${ }^{18}$ approximates the self energy of the original system in the thermodynamic limit by the self energy of an exactly solvable reference system with the same interaction part. The self energy is varied by varying the single-particle parameters of the reference system. Choosing the reference system to be a cluster of finite size yields a non-perturbative and consistent cluster approach. It has been shown ${ }^{19}$ that within this framework the CPT as well as the C-DMFT appear as special approaches depending on the number of additional uncorrelated ("bath") sites taken into account: The optimum number of bath sites is actually a free parameter which can be determined from the general variational principle. It has been pointed out ${ }^{19}$ that at least for one-dimensional models a large cluster without bath sites has to be preferred. The use of a reference system without bath sites represents a generalized CPT in which the single-particle parameters of the finite cluster are optimized according to the variational principle. This "variational CPT" (V-CPT) has successfully been used in a recent study for the investigation of the symmetrybroken antiferromagnetic phase of the two-dimensional Hubbard model. 20

So far a consistent formulation of the (variational) cluster-perturbation approach could be achieved for lattice models with on-site interactions only. The reason for this restriction is that within the SFA the reference system must be chosen with the same interaction as the original model. As detailed in Ref.18, this ensures that functionals given by the skeleton-diagram expansion are the same for both, the original and the reference model. In case of the EHM the interaction couples the different sites of the lattice. Thus there is no reference system with the same interaction which consists of decoupled subsystems of finite size. The motivation of the present paper is therefore to extend the ideas of the CPT and V-CPT to the investigation of the EHM including nearest-neighbor Coulomb interaction. It is shown that a mean-field decoupling of the inter-cluster nearest-neighbor interaction yields a systematic and reliable cluster approach.

The paper is organized as follows: In Sec.II we give a short description of the V-CPT method, Sec.III shows how to decouple clusters in the case of the EHM. In Sec.[IV and Sec. $[\nabla$ we present results for one and two dimensions, respectively. The conclusions are given in Sec.VI

\section{VARIATIONAL CPT}

Let us consider a system of interacting fermions on a lattice with Hamiltonian $H$, in general consisting of a single-particle part $H_{0}$ and an interaction part $H_{1}$. The lattice is then divided into clusters, where it is of crucial importance for the derivation of the method that those clusters are connected by $H_{0}$ only. The Hamiltonian can then be written as

$$
H=\sum_{\mathbf{R}}\left[H_{0}^{(\mathrm{c})}(\mathbf{R})+H_{1}(\mathbf{R})\right]+\sum_{\mathbf{R}, \mathbf{R}^{\prime}} H_{0}^{(\mathrm{i})}\left(\mathbf{R}, \mathbf{R}^{\prime}\right),
$$

where $\mathbf{R}$ denotes the individual clusters, $H_{0}^{(\mathrm{c})}(\mathbf{R})$ is the part of the single-particle term that acts only inside a single cluster, $H_{1}(\mathbf{R})$ is the interaction part inside the cluster, and the inter-cluster hopping is given by

$$
H_{0}^{(\mathrm{i})}\left(\mathbf{R}, \mathbf{R}^{\prime}\right)=\sum_{a, b} T_{a, b}^{\mathbf{R}, \mathbf{R}^{\prime}} c_{\mathbf{R}, a}^{\dagger} c_{\mathbf{R}^{\prime}, b},
$$

where the hopping matrix $T_{a, b}^{\mathbf{R}, \mathbf{R}^{\prime}}$ is non-zero only for hopping processes across the cluster boundaries. The indices $a$ and $b$ are general quantum numbers within a cluster, e.g. position and spin index, and $c_{\mathbf{R}, a}^{\dagger}$ creates an electron with quantum number $a$ in cluster $\mathbf{R}$.

The quantity of interest is the single particle Green's function $G_{\mathbf{R}, a, \mathbf{R}^{\prime}, b}(\omega)=\left\langle\left\langle c_{\mathbf{R}, a} ; c_{\mathbf{R}^{\prime}, b}^{\dagger}\right\rangle\right\rangle_{\omega}$. Using translational invariance at the level of the superlattice vector $\mathbf{R}$, the Green's function becomes diagonal with respect to the wave vector $\mathbf{Q}$ from the reduced Brillouin zone corresponding to the superlattice. The resulting Green's function in reciprocal space is a matrix $\mathbf{G}_{\mathbf{Q}}(\omega)$ with elements $G_{\mathbf{Q}, a, b}(\omega)$ and $a, b$ quantum numbers within a cluster.

Within the CPT approximation this Green's function $\mathbf{G}_{\mathbf{Q}}(\omega)$ can be expressed in terms of Green's functions of the decoupled clusters $\mathbf{G}^{\prime}(\omega)$, again matrices in the quantum numbers $a$ and $b$, and the inter-cluster hopping $T_{a, b}^{\mathbf{R}, \mathbf{R}^{\prime}}$ by the expression

$$
\mathbf{G}_{\mathbf{Q}}(\omega)=\left[\mathbf{G}^{\prime}(\omega)^{-1}-\mathbf{T}_{\mathbf{Q}}\right]^{-1}
$$

with the Fourier-transformed inter-cluster hopping

$$
T_{\mathbf{Q}, a, b}=\frac{1}{L} \sum_{\mathbf{R}, \mathbf{R}^{\prime}} T_{a, b}^{\mathbf{R}, \mathbf{R}^{\prime}} e^{i \mathbf{Q}\left(\mathbf{R}-\mathbf{R}^{\prime}\right)} .
$$

For the details of the derivation of the CPT formulas we refer the interested reader to Refs. 1213 and references therein. We want to mention that one can transform Eq. (3) into a Dyson-like equation

$$
\mathbf{G}_{\mathbf{Q}}(\omega)=\left(\mathbf{G}_{\mathbf{Q}}^{(0)}(\omega)^{-1}-\boldsymbol{\Sigma}(\omega)\right)^{-1},
$$

where $\mathbf{G}_{\mathbf{Q}}^{(0)}(\omega)$ is the free Green's function of the infinite lattice, and $\boldsymbol{\Sigma}(\omega)$ is the cluster self energy. In other words CPT consists of approximating the self energy of the infinite system by the self energy of a cluster of finite size. Note that CPT is based on the exact evaluation of small clusters without any self-consistency procedure, and thus does not allow for the occurrence of symmetrybroken phases. This restriction is overcome with the $\mathrm{V}$ CPT method 19,20 
The observation underlying $\mathrm{V}-\mathrm{CPT}$ is that the perturbation term need not necessarily be restricted to the inter-cluster hopping term but can be any single-particle operator. For this reason one has a certain amount of freedom for the partitioning of the single-particle part of Eq. (11). Namely, the Hamiltonian Eq. (11) is invariant under the transformation

$$
\begin{aligned}
H_{0}^{(\mathrm{c})}(\mathbf{R}) & \rightarrow H_{0}^{(\mathrm{c})}(\mathbf{R})+\mathcal{O}(\mathbf{R}) \\
H_{0}^{(\mathrm{i})}\left(\mathbf{R}, \mathbf{R}^{\prime}\right) & \rightarrow H_{0}^{(\mathrm{i})}\left(\mathbf{R}, \mathbf{R}^{\prime}\right)-\delta_{\mathbf{R}, \mathbf{R}^{\prime}} \mathcal{O}(\mathbf{R}),
\end{aligned}
$$

with an arbitrary intra-cluster single-particle operator

$$
\mathcal{O}(\mathbf{R})=\sum_{a, b} \Delta_{a, b} c_{\mathbf{R}, a}^{\dagger} c_{\mathbf{R}, b}
$$

which can for instance be a fictitious symmetry-breaking field, thus allowing for broken symmetry already on a finite system instead of only in the thermodynamic limit. In the non-interacting case, where Eq. (3) is exact, the result is independent of the transformation Eq. ([6), but in the interacting case the result depends on the particular choice. However, this dependence is not a shortcoming of the method but can rather be used for an optimization procedure.

The question of what choice for $\boldsymbol{\Delta}=\Delta_{a, b}$ will optimize the results can be answered by the SFA $\underline{18}$ It provides a way to exactly evaluate the grand potential $\Omega[\boldsymbol{\Sigma}]$ as functional of the self-energy $\boldsymbol{\Sigma}$ by restricting the domain of the functional to a certain subspace $\mathcal{S}$ of trial self-energies. This subspace consists of all $\boldsymbol{\Sigma}$ which are exact self-energies of the reference system for different $\boldsymbol{\Delta}$. The reference system $H^{\prime}$ must have the same interaction part as $H$ and must be exactly solvable for any $\boldsymbol{\Delta}$. Throughout the paper we use a cluster of finite size as reference system.

The cluster self-energy can be parametrized as $\boldsymbol{\Sigma}=$ $\boldsymbol{\Sigma}(\boldsymbol{\Delta})$. Within the SFA, the optimal value of $\boldsymbol{\Delta}$ is determined from the stationary point of the function ${ }^{18}$

$$
\begin{aligned}
\Omega(\boldsymbol{\Delta}) & =\Omega^{\prime}(\boldsymbol{\Delta}) \\
& +T \sum_{\omega_{n}, \mathbf{Q}} \operatorname{tr} \ln \frac{-\mathbf{1}}{\mathbf{G}_{\mathbf{Q}}^{(0)}\left(i \omega_{n}\right)^{-1}-\mathbf{\Sigma}\left(\boldsymbol{\Delta}, i \omega_{n}\right)} \\
& -L T \sum_{\omega_{n}} \operatorname{tr} \ln \left(-\mathbf{G}^{\prime}\left(\boldsymbol{\Delta}, i \omega_{n}\right)\right),
\end{aligned}
$$

where $\Omega^{\prime}(\boldsymbol{\Delta})$ is the grand potential of the reference system. The frequency sum runs over discrete Matsubara frequencies $i \omega_{n}, L$ is the number of clusters or $\mathbf{Q}$ points, respectively, $T$ gives the temperature, and bold symbols denote matrices in the cluster indices $a$ and $b$. Note that the fraction in the second line in Eq. (8) is the CPT Green's function. The single-particle parameters $\boldsymbol{\Delta}$ can include all single-particle parameters of the original Hamiltonian or only part of it, as well as additional terms, e.g. a fictitious staggered field. The more parameters are considered for the optimization problem, the larger is the subspace $\mathcal{S}$ of trial self energies. The actual choice and number of parameters depends on the problem under consideration. For more details of the derivation of the method see Ref.20. Note that the V-CPT method is superior to the CPT approach, since within the concept of V-CPT one gets the standard CPT formulas by setting the number of variational parameters $\boldsymbol{\Delta}$ to zero.

A necessary condition for the applicability of the method is that the clusters are coupled by single-particle operators only. At this point it is easy to see that a straightforward application of the method to the EHM where the clusters are also coupled by Coulomb interactions is not possible. However, we will show in Sec.III how one can decouple the lattice into clusters appropriate for the application of CPT even in the case of the EHM.

\section{DECOUPLING THE CLUSTERS}

We start from the Hamiltonian of the extended Hubbard model

$$
\begin{aligned}
H & =\sum_{i j, \sigma} T_{i, j} c_{i \sigma}^{\dagger} c_{j \sigma}+U \sum_{i} n_{i \uparrow} n_{i \downarrow} \\
& +V \sum_{\langle i j\rangle} n_{i} n_{j}-\mu \sum_{i} n_{i},
\end{aligned}
$$

where $i, j$ indicate the position in the lattice, and for convenience we use a constant value $V_{i, j} \equiv V$ for all nearestneighbor bonds. According to Eq. (1) we decouple the lattice into clusters yielding

$$
\begin{aligned}
H & =\sum_{\mathbf{R}}\left[H_{0}^{(\mathrm{c})}(\mathbf{R})+H_{\mathrm{U}}^{(\mathrm{c})}(\mathbf{R})+H_{\mathrm{V}}^{(\mathrm{c})}(\mathbf{R})\right] \\
& +\sum_{\mathbf{R}, \mathbf{R}^{\prime}}\left[H_{0}^{(\mathrm{i})}\left(\mathbf{R}, \mathbf{R}^{\prime}\right)+H_{\mathrm{V}}^{(\mathrm{i})}\left(\mathbf{R}, \mathbf{R}^{\prime}\right)\right],
\end{aligned}
$$

where the first row includes only terms of a single cluster and the second row couples different clusters. By comparing the second row with the corresponding term in Eq. (11) one can see that the term causing problems in the case of the EHM is the interaction term

$$
H_{V}^{(\mathrm{i})}\left(\mathbf{R}, \mathbf{R}^{\prime}\right)=V \sum_{[i j]} n_{\mathbf{R} i} n_{\mathbf{R}^{\prime} j},
$$

which is of two-particle type. The symbol $[i j]$ indicates that the sum runs only over bonds connecting nearest neighbors in different clusters. For nearest-neighbor interactions this means that the indices in $[i j]$ must belong to the cluster boundaries of two adjacent clusters. For the application of the method derived in Sec.II the coupling term must be of single-particle type, which can be achieved by a mean-field decoupling of the interaction term Eq. (11). Hence we get

$$
\begin{aligned}
H_{\mathrm{V}, \mathrm{MF}}^{(\mathrm{i})}\left(\mathbf{R}, \mathbf{R}^{\prime}\right) & =V \sum_{[i j]}\left[n_{\mathbf{R}_{i} i}\left\langle n_{\mathbf{R}^{\prime} j}\right\rangle+\left\langle n_{\mathbf{R}_{i} i}\right\rangle n_{\mathbf{R}^{\prime} j}\right] \\
& -V \sum_{[i j]}\left\langle n_{\mathbf{R}_{i}}\right\rangle\left\langle n_{\mathbf{R}^{\prime} j}\right\rangle .
\end{aligned}
$$


Due to the translational invariance with respect to the superlattice vector $\mathbf{R}$, the mean-field parameters $\left\langle n_{\mathbf{R} i}\right\rangle$ and $\left\langle n_{\mathbf{R}^{\prime} j}\right\rangle$ are independent of $\mathbf{R}$ and $\mathbf{R}^{\prime}$ and will be denoted by $\lambda_{i}$ and $\lambda_{j}$, respectively. With these abbreviations we get

$$
\begin{aligned}
\sum_{\mathbf{R}, \mathbf{R}^{\prime}} & H_{\mathrm{V}, \mathrm{MF}}^{(\mathrm{i})}\left(\mathbf{R}, \mathbf{R}^{\prime}\right)= \\
& =V \sum_{\mathbf{R}, \mathbf{R}^{\prime}} \sum_{[i j]}\left[n_{\mathbf{R} i} \lambda_{j}+n_{\mathbf{R}^{\prime} j} \lambda_{i}-\lambda_{i} \lambda_{j}\right] \\
& =V \sum_{\mathbf{R}} \sum_{[i j]}\left[n_{\mathbf{R} i} \lambda_{j}+n_{\mathbf{R}_{j}} \lambda_{i}-\lambda_{i} \lambda_{j}\right] \\
& =\sum_{\mathbf{R}} H_{\mathrm{V}, \mathrm{MF}}^{(\mathrm{i})}(\mathbf{R}) .
\end{aligned}
$$

The double sum over $\mathbf{R}$ and $\mathbf{R}^{\prime}$ reduces to a single sum, because for fixed values of $\mathbf{R}, i$, and $j$ only one term of the sum over $\mathbf{R}^{\prime}$ contributes due to the fact that two-site interactions couple at most two different clusters. One has to be careful in order to avoid double counting of the bonds $[i j]$. For instance, for a one-dimensional cluster of length $N$, Eq. (13) reduces to

$$
V \sum_{\mathbf{R}}\left[n_{\mathbf{R} 1} \lambda_{N}+n_{\mathbf{R} N} \lambda_{1}-\lambda_{1} \lambda_{N}\right],
$$

because the only decoupled bond connects sites 1 and $N$ of different clusters.

By this mean-field decoupling, two parameters $\lambda_{i}$ are introduced for each decoupled bond, e.g. $\lambda_{1}$ and $\lambda_{N}$ in one dimension, and in general all these parameters $\lambda_{i}$ are independent of each other. But as we will see below, the number of mean-field parameters $\lambda_{i}$ can be strongly reduced in special cases.

The decoupled interaction Eq. (13) is of single-particle type and can be included in the intra-cluster hopping term $H_{0}^{(\mathrm{c})}(\mathbf{R})$, leading to a modified intra-cluster singleparticle term

$$
\tilde{H}_{0}^{(\mathrm{c})}\left(\mathbf{R}, \lambda_{i}\right)=H_{0}^{(\mathrm{c})}(\mathbf{R})+H_{\mathrm{V}, \mathrm{MF}}^{(\mathrm{i})}\left(\mathbf{R}, \lambda_{i}\right),
$$

where we explicitly denoted the dependence on the parameters $\lambda_{i}$. After mean-field decoupling we finally get the Hamiltonian

$$
\begin{aligned}
H_{\mathrm{MF}}\left(\lambda_{i}\right) & =\sum_{\mathbf{R}}\left[\tilde{H}_{0}^{(\mathrm{c})}\left(\mathbf{R}, \lambda_{i}\right)+H_{\mathrm{U}}^{(\mathrm{c})}(\mathbf{R})+H_{\mathrm{V}}^{(\mathrm{c})}(\mathbf{R})\right] \\
& +\sum_{\mathbf{R}, \mathbf{R}^{\prime}} H_{0}^{(\mathrm{i})}\left(\mathbf{R}, \mathbf{R}^{\prime}\right),
\end{aligned}
$$

for which the method described in Sec. II is applicable.

From the decoupling of the clusters we have got additional parameters $\lambda_{i}$ which are external parameters to the Hamiltonian Eq. (16) and have to be determined in a proper way. For this purpose we propose two different procedures:

(i) One can get the parameters from a self-consistent calculation on an isolated cluster. That means that one starts with a certain guess for the $\lambda_{i}$, which are the expectation values of the electron densities on sites $i$. Then the ground-state wave function of an isolated cluster is calculated, giving new values for the $\lambda_{i}$. In this step open boundary conditions (obc) are used in order to be consistent with the obc necessary for the calculation of the cluster Green's function in Eqs. (3) and (8). These new values $\lambda_{i}$ serve as parameters in the Hamiltonian for the next determination of the ground state, and the whole procedure is iterated until convergence of the $\lambda_{i}$ is achieved. This procedure may work quite well for the EHM in the case of a first order phase transition between a disordered and an ordered phase, because (due to an avoided level crossing) the transition point, i.e. the critical Coulomb interaction $V_{c}$, is almost independent of the cluster size ${ }^{21}$ For second order phase transitions we expect that this method will not give satisfactory results, because here we face a discrepancy between the parameters calculated on the isolated cluster and the parameters that would give the optimal result in the thermodynamic limit.

(ii) The shortcoming in the case of second order phase transitions can be overcome in the following way: As we show in App. A the self-consistent calculation of meanfield parameters is equivalent to the minimization of the free energy $F$. Since the relation $\Omega=F-\mu N$ holds at $T=0$, this minimization can be done at the same time as the optimization of the single-particle parameters $\boldsymbol{\Delta}$ in the SFA formalism, and we can use Eq. (8) for the determination of the parameters $\lambda_{i}$, too. Note that all quantities in Eq. (8) which depend on the single-particle parameters $\boldsymbol{\Delta}$ are dependent on the mean-field parameters $\lambda_{i}$ as well. To keep the calculations simple we consider only half-filled systems, where it is sufficient to use only two different values for the $\lambda_{i}$, namely $\lambda_{A}=1-\delta$ and $\lambda_{B}=1+\delta$ on sublattices $A$ and $B$, respectively. Under this assumption we have only one mean-field parameter $\delta$, and the grand potential is $\Omega=\Omega(\boldsymbol{\Delta}, \delta)$. The general procedure is now, that for each value of $\delta$ the stationary point with respect to $\Delta$ has to be found as required by the SFA formalism, yielding a function $\Omega=\Omega(\delta)$. By finding the minimum of this function one can determine the optimal value for $\delta$.

Conceptually, the latter method (ii) of determining the mean-field parameters is superior to the procedure (i) described first as it uses information on the Green's function in the thermodynamic limit for the calculation of $\delta$. However, one has to keep in mind that for each choice of $\delta$ the Green's function $\mathbf{G}^{\prime}(\omega)$ of the isolated cluster has to be calculated many times to evaluate Eq. (8) which is much more time consuming than the self-consistency procedure on the isolated cluster. 


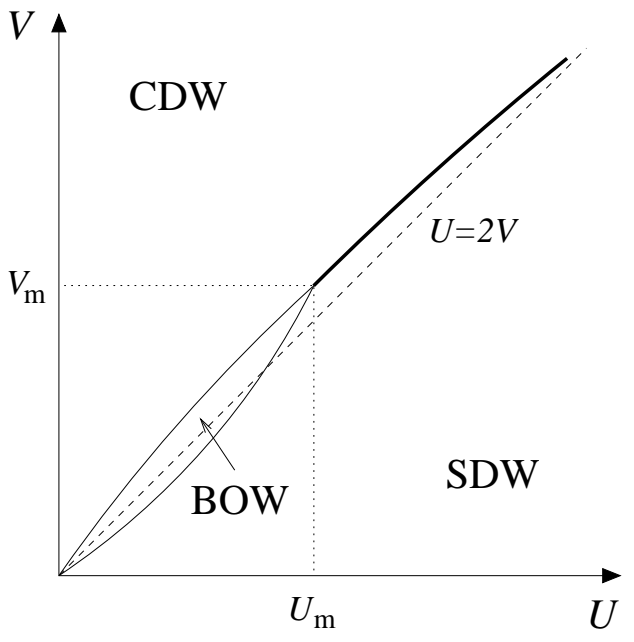

FIG. 1: Schematic phase diagram of the one-dimensional EHM taken from Refs. 212527 . The thick line marks the first order phase transition, and the dashed line marks $U=2 \mathrm{~V}$.

\section{ONE DIMENSION} by

The Hamiltonian of the one-dimensional EHM is given

$$
\begin{aligned}
H= & -t \sum_{i, \sigma}\left(c_{i, \sigma}^{\dagger} c_{i+1, \sigma}+\text { H.c. }\right)+U \sum_{i} n_{i \uparrow} n_{i \downarrow} \\
& +V \sum_{i} n_{i} n_{i+1}-\mu \sum_{i} n_{i} .
\end{aligned}
$$

Throughout the paper we set $t$ as the unit of energy. Although this model was studied intensively in recent years, the ground state phase diagram is still under some discussion $21,22,23,24,25,26,27$ We use this model as a testing ground for our method, because many results are available for comparison. The chemical potential is $\mu=U / 2+2 V$ due to particle-hole symmetry at halffilling.

In one dimension at half filling, the EHM shows an involved phase diagram including spin density wave (SDW), charge density wave (CDW), and bond order wave (BOW) phases as shown schematically in Fig.1 There is good agreement on the existence of the latter phase, but its extension in the $U-V$-plane has not yet been clarified in detail.21.26 Moreover, a multi-critical point occurs at some critical value $U_{m}$, where the phase transition into the ordered CDW phase changes from a second order transition at lower values to a first order transition at higher values. Recent QMC studies 21 gave $U_{m} \approx 4.7$ for this point in good agreement with

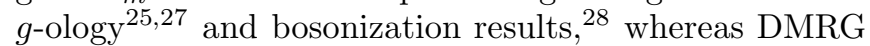
gives a somewhat lower value $U_{m} \approx 3.7 \underline{26}$

\section{A. First order phase transition}

For a first test of our method we studied the onedimensional EHM at $U=8$, which is well above the multi-critical point. The phase transition is then of first order without any BOW phase between SDW and CDW phases. As reference system $H^{\prime}$ according to Sec.III we used decoupled clusters of different lengths consisting of $N_{c}=8,10$, and 12 sites, respectively. For the determination of the mean-field parameter $\delta$ we used the method (ii) described in Sec.III where $\delta$ is calculated from the minimum of the free energy of the system. For the SFA optimization of the single-particle parameters, we had to choose a set $\boldsymbol{\Delta}$ of parameters which are varied in the optimization procedure. In order to minimize the number of relevant parameters we used results of a recent study of the Hubbard model ${ }^{19}$ There it has been shown that at $U=8$ the variation of the hopping in the cluster yields only minor changes that can be neglected, and that open boundary conditions have to be used. Moreover, for the one-dimensional Hubbard model it has been pointed out that the use of a fictitious staggered magnetic field as a variational parameter gives a stationary point of the grand potential only for vanishing field, yielding a paramagnetic phase without long-range magnetic order. Since it can be assumed that these results are also valid in a similar way for the EHM, we did not use the hopping in the cluster or a staggered magnetic field in the optimization procedure. Here we studied charge-ordering effects and therefore we used as variational parameter a staggered field coupled to the charge densities given by Eq. (7) with

$$
\Delta_{a, b}=\varepsilon \delta_{a, b} e^{i \mathbf{Q R} \mathbf{R}_{a}},
$$

where $\mathbf{Q}=\pi$ is the wave vector of staggered ordering and $\varepsilon$ is the staggered-field strength. The grand potential obtained in this way is shown in Fig.2 at two values of the inter-site Coulomb interaction. For comparison, calculations without optimization of the staggered field are shown as dashed lines in Fig.2 As one can see, the optimization gives only minor changes to $\Omega(\delta)$. The optimal staggered-field strengths in these calculations varied between $\varepsilon_{\mathrm{opt}}=0.0$ at $\delta=0.0$ and $\varepsilon_{\mathrm{opt}} \approx 0.05$ at $\delta=1.0$ at both values of $V$.

From the shape of $\Omega(\delta)$ one can directly infer the order of the transition. If three minima occur at $\delta=0$ and $\delta= \pm \delta_{\mathrm{CDW}}$, it is of first order, whereas it is of second order if $\Omega(\delta)$ has only two minima at $\delta= \pm \delta_{\mathrm{CDW}}$ and a maximum at $\delta=0$. As on can easily see in Fig.2 we have clear evidence for a first order phase transition at $U=8$ with an SDW minimum at $\delta=0.0$ and two degenerate CDW minima at $\delta= \pm \delta_{\mathrm{CDW}}$. At $V=4.1$ the SDW phase is realized, $\Omega(0)<\Omega\left(\delta_{\mathrm{CDW}}\right)$, whereas at $V=4.2$ we have $\Omega(0)>\Omega\left(\delta_{\mathrm{CDW}}\right)$ and the CDW phase is the stable one. Thus we can state that the critical value $V_{c}$ for the phase transition is located between $V=4.1$ and $V=4.2$. 


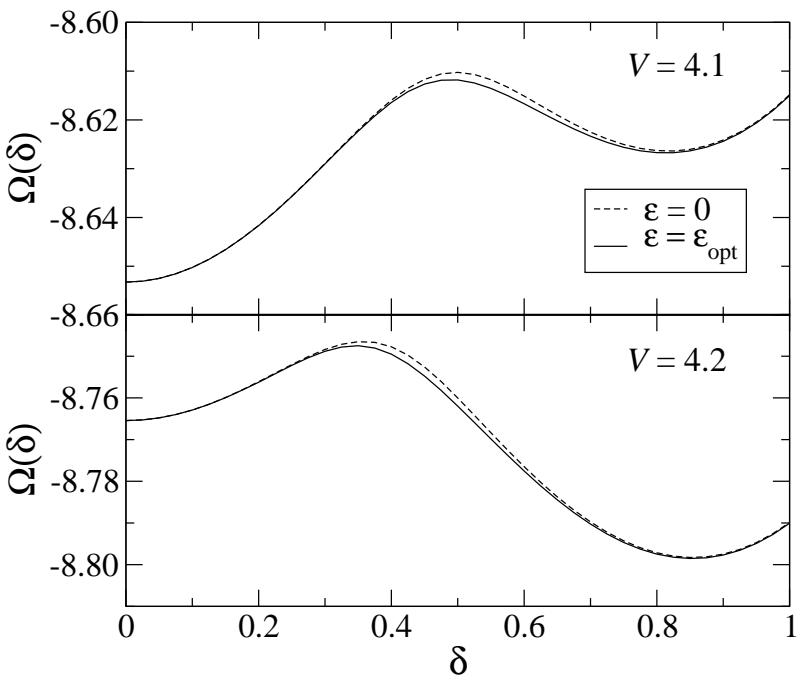

FIG. 2: Grand potential $\Omega$ as a function of the mean-field parameter $\delta$ at $U=8$ calculated on a cluster with $N_{c}=8$ sites as reference system. Upper panel: $V=4.1$. Lower panel: $V=4.2$. Solid lines: With optimization of a staggered field. Dashed lines: Without optimization of a staggered field.

For a more accurate determination of the phase boundary $V_{c}$, we have calculated the grand potential at several values of $V$ and cluster sizes $N_{c}=8,10$, and 12 . In addition to the grand potential and the ground state energy $E_{0}=\Omega+\mu N_{e}$ with $N_{e}$ the number of electrons in the system, we calculated the order parameter

$$
m_{\mathrm{CDW}}=\frac{1}{N_{c}} \sum_{j}\left(n_{j}-\langle n\rangle\right) e^{i \mathbf{Q R}_{j}}
$$

where $\mathbf{Q}=\pi, N_{c}$ is the number of cluster sites, and the kinetic energy $E_{\text {kin }}$. Both properties can be extracted from the spectral function $A_{\mathbf{R}, \mathbf{r}, \mathbf{R}^{\prime}, \mathbf{r}^{\prime}, \sigma}(\omega)=$ $(-1 / \pi) \operatorname{Im}\left\langle\left\langle c_{\mathbf{R}, \mathbf{r}, \sigma} ; c_{\mathbf{R}^{\prime}, \mathbf{r}^{\prime}, \sigma}^{\dagger}\right\rangle\right\rangle_{\omega}^{(\text {ret })}$. The kinetic energy is obtained after Fourier-transformation ${ }^{12.13 .20}$

$$
A(\mathbf{k}, \omega)=\frac{1}{L N_{c}} \sum_{\mathbf{R}, \mathbf{R}^{\prime}} \sum_{\mathbf{r}, \mathbf{r}^{\prime}} e^{i \mathbf{k}\left(\mathbf{R}+\mathbf{r}-\mathbf{R}^{\prime}-\mathbf{r}^{\prime}\right)} A_{\mathbf{R}, \mathbf{r}, \mathbf{R}^{\prime}, \mathbf{r}^{\prime}, \sigma}(\omega)
$$

by

$$
E_{\text {kin }}=\frac{2}{L} \sum_{\mathbf{k}} \int_{-\infty}^{0} \mathrm{~d} \omega \varepsilon(\mathbf{k}) A(\mathbf{k}, \omega),
$$

with $\varepsilon(\mathbf{k})$ the dispersion of the non-interacting system. Within our approach it is necessary to use the Lehmann representation for the cluster Green's function with small but finite Lorentzian broadening $\sigma$. Whereas the grand potential Eq. (8) shows only minor dependence on this broadening, the dependence of the order parameter and the kinetic energy is considerably larger and one has to do an extrapolation to $\sigma=0 . \frac{13}{13}$ Although the formalism applies to the thermodynamic limit, results show a

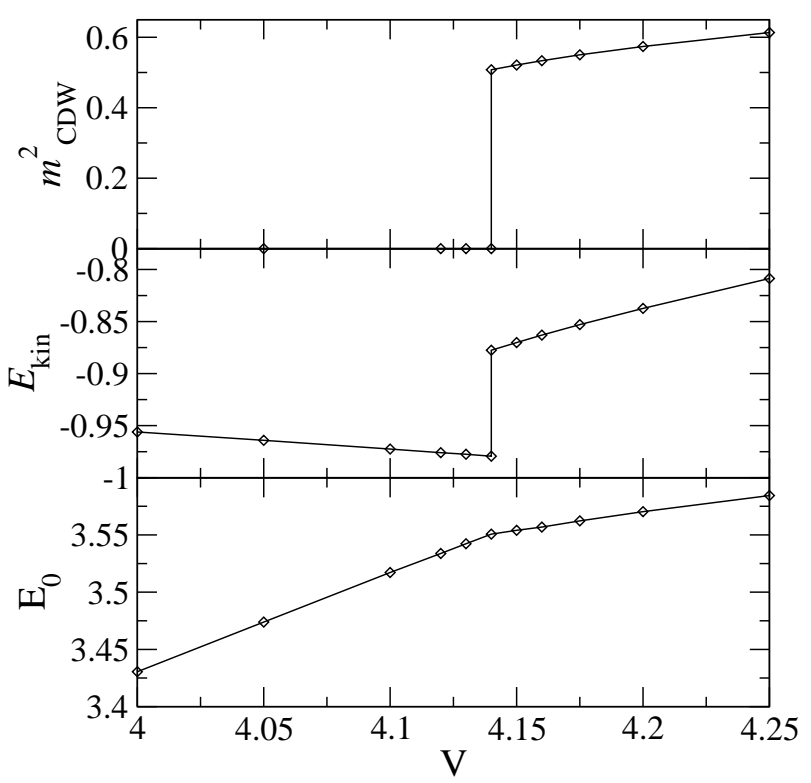

FIG. 3: Ground state energy $E_{0}$, kinetic energy $E_{\text {kin }}$, and order parameter $m_{\mathrm{CDW}}^{2}$ of the one-dimensional EHM at $U=8$ after finite size scaling. Lines are guides to the eye only.

finite size dependence due to the finite size of the clusters serving as reference system. We found that the order parameter exhibits the strongest finite-size effects, which were of the order $m_{\mathrm{CDW}, N_{c}=10}^{2} / m_{\mathrm{CDW}, N_{c}=12}^{2} \approx 1.02$ at all values of $V$. Finite-size scaling to $N_{c}=\infty$ is easily done and the results for the ground state energy extracted from the minimum of the grand potential, the kinetic energy and the order parameter are shown in Fig. 3. Our results should be compared to Fig. 10 of Ref.21 which shows excellent quantitative agreement with a deviation of less than $2 \%$ for the calculated quantities at all values of $V$. From our calculations we get $V_{c}=4.140(5)$, again in agreement with the previous studies Refs. 2126.

In order to provide a complete picture of the method we also performed calculations with mean-field parameters obtained by a self-consistent procedure on an isolated cluster, see method (i) in Sec.III For instance for $N_{c}=12$ and $V=4.1$ one finds self-consistent solutions for $\delta=0$ and for $\delta_{\mathrm{SC}}=0.832$, which differs only slightly from the value extracted from the grand potential, $\delta_{\mathrm{CDW}}=0.822$. For this reason the calculation of the ground-state energy, kinetic energy, and order parameter using $\delta_{\mathrm{SC}}$ instead of $\delta_{\mathrm{CDW}}$ gives practically the same results as in Fig. 3. In the present case it is therefore sufficient to calculate the mean-field parameter from an isolated cluster which is much faster than finding the minimum of the grand potential.

Whereas the properties we have shown so far are well known for the one-dimensional EHM, we additionally calculated for the first time the spectral function by Eq. (20) for arbitrary wave vector $\mathbf{k}$. In Fig. 4 results are shown at $U=8$ and selected values of $V$ with a reference system consisting of $N_{c}=12$ cluster sites, and the mean-field 

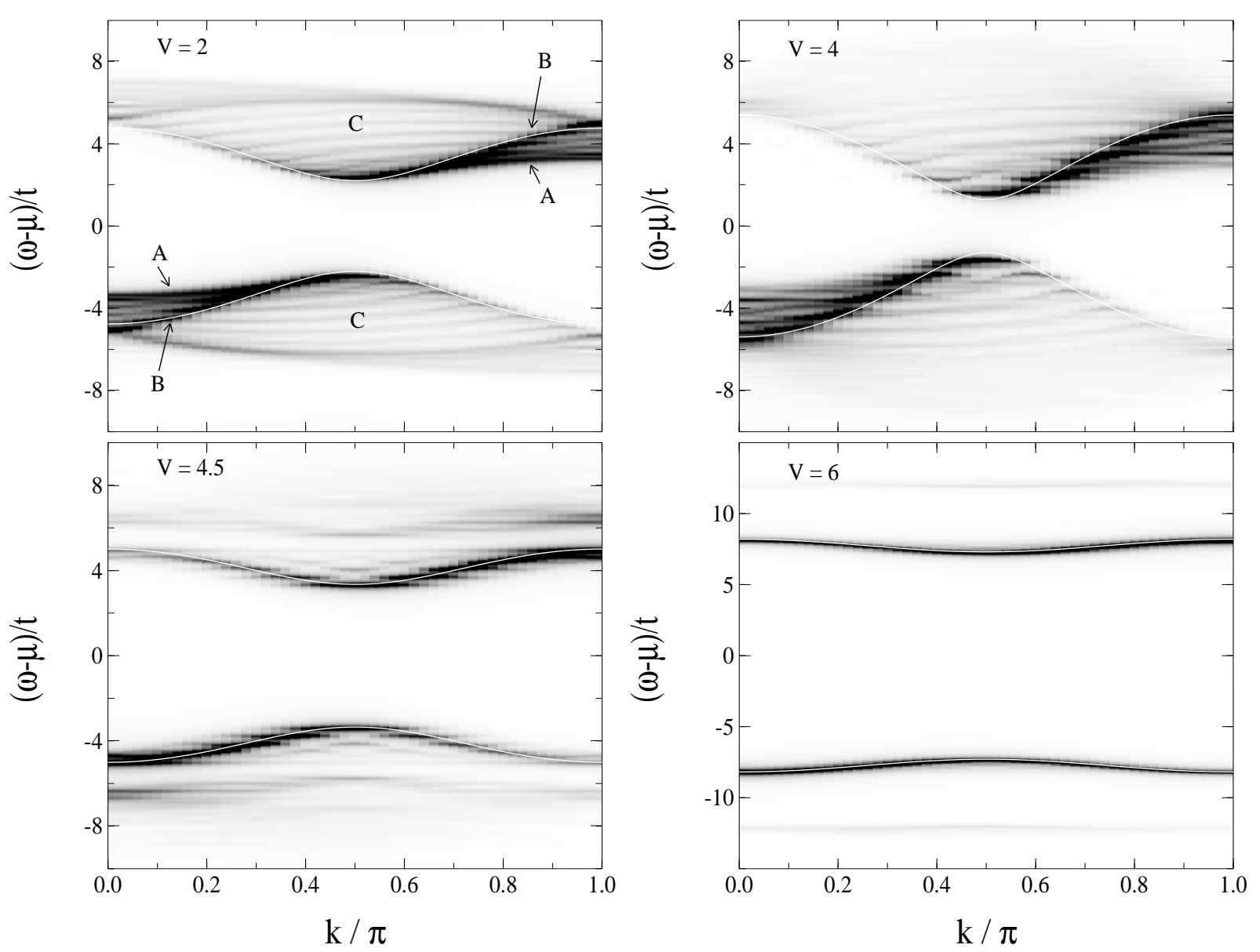

FIG. 4: Density plot of the spectral function $A(\mathbf{k}, \omega)$ of the one-dimensional EHM at $U=8$, calculated on a cluster of size $N_{c}=12$ with Lorentzian broadening $\sigma=0.1$. Darker regions represent larger spectral weight. Coulomb interaction $V$ as indicated in the plots. White lines are fits to a Hartree-Fock SDW/CDW dispersion (see text).

parameter $\delta$ calculated self-consistently by method (i), see Sec.III] We want to mention that the 'striped' structure, particularly visible in the regions marked by 'C' in Fig. [4 occurs because the decoupling into clusters breaks the translational invariance of the system.

The spectral function at $V=2.0$ is very similar to the spectral function of the Hubbard model $(V=0)^{12.13}$ with splitting of the low-energy band into a spinon and an holon band, which are marked in Fig. 4 by 'A' and 'B', respectively. This similarity could have already been expected based on the full Hartree-Fock solutiondecoupling of all interaction terms in the Hamiltonianwhere one has no dependence on $V$ at all in the SDW phase. But this simple picture holds only away from the transition point $V_{c}$ as can be seen in Fig. 4 in the plot at $V=4.0$. At this point, in the vicinity of the phase transition $V_{c}=4.14$, the gap is considerably smaller than at $V=2.0$, a clear deviation from the Hartree-Fock prediction. This indicates that charge fluctuations become very important in this regime, which are completely neglected by the Hartree-Fock approximation, but are taken into account on the length scale of the cluster in our approach.
TABLE I: Fitted values for the hopping matrix element $t_{\text {fit }}$, gap $\Delta_{\text {fit }}$, and gap $\Delta_{\mathrm{HF}}$ of the full Hartree-Fock approximation at $U=8$.

\begin{tabular}{|c|c|c|c|}
\hline & $t_{\text {fit }}$ & $\Delta_{\text {fit }}$ & $\Delta_{\mathrm{HF}}$ \\
\hline$V=0.0$ & 1.93 & 2.24 & 3.75 \\
$V=2.0$ & 2.11 & 2.20 & 3.75 \\
$V=4.0$ & 2.62 & 1.29 & 3.75 \\
$V=4.5$ & 1.86 & 3.35 & 4.80 \\
$V=6.0$ & 1.86 & 7.29 & 7.88 \\
\hline
\end{tabular}

But although we found this deviation, one can still see residuals of the splitting of the low-energy band, a signature for spin-charge separation. For this reason we infer that spin-charge separation is present up to the transition point. The white lines in Fig. [4 correspond to fits of the holon branch to a Hartree-Fock dispersion $E(\mathbf{k})= \pm \sqrt{\Delta^{2}+\varepsilon(\mathbf{k})^{2}}$. The fitted values for the hopping matrix element $t_{\text {fit }}$ and the gap $\Delta_{\text {fit }}$ are denoted in Tab. I where we included the values at $V=0$ for completeness. One finds that the gap $\Delta_{\text {fit }}$ is almost constant 
from $V=0$ to $V=2$ and, as mentioned above, considerably decreases near the the phase transition $(V=4)$. The hopping matrix element $t_{\text {fit }}$ shows the opposite behavior and increases when approaching the transition point from below. This is due to the fact that in the vicinity of $V_{c}$, doubly-occupied and singly-occupied sites become close in energy, which enhances the movement of the electrons. The actual value of the matrix element $t_{\text {fit }}$ is very large compared to the original value $t=1$ in the Hamiltonian. A fit to the spinon band would give a smaller value closer to $t=1$, but whereas fitting to the holon band is consistent over the whole range of momentum vectors $\mathbf{k}$, the spinon band is only present for $\mathbf{k}<\pi / 2$ for $\omega-\mu<0$ (and $\mathbf{k}>\pi / 2$ for $\omega-\mu>0$, respectively).

The spectral function in the CDW phase shows a qualitatively different behavior. At $V=4.5$ we found a gap considerably larger than in the SDW phase, and this gap increases very fast with increasing $V$, as can be seen in the plot at $V=6$. Moreover, no evidence for spin-charge separation can be seen in the spectral functions. By comparing the fitted value $\Delta_{\text {fit }}$ with the Hartree-Fock solution $\Delta_{\mathrm{HF}}$, one can see that the agreement at $V=4.5$ is better than at $V=4$, and that it becomes still better with increasing $V$. For this reason we conclude that charge fluctuations which are neglected in the HartreeFock approximation play a minor role in the CDW phase.

\section{B. Second order phase transition}

So far all calculations were done at $U=8$, where the system shows a first order phase transition. In the following, we study the EHM at $U=3$, where the model exhibits a second order transition into the charge ordered CDW phase ${ }^{21.26}$ In this paper we do not consider the BOW, since it has been argued that the SDW-BOW transition is of Kosterlitz-Thouless type ${ }^{24}$ For an analysis of this type of transition the available cluster sizes are far too small and do not allow a clear distinction between SDW and BOW phase.

We calculate the grand potential $\Omega(\delta)$ in the same way as in Sec.IVA in order to determine $\delta$. The result of a calculation on a cluster consisting of $N_{c}=8$ sites is shown in Fig. 5 One can easily see a striking difference between the grand potential at $U=8$, Fig. 22 and at $U=3$. In the latter case there is only a single minimum. It is located at $\delta=0$ for $V<V_{c}$. With increasing $V$ the curve for $\Omega(\delta)$ becomes flatter in the region around $\delta=0$ and finally two degenerate CDW minima occur at $\delta= \pm \delta_{\mathrm{CDW}}$ for $V>V_{c}$. Note that here $\delta$ changes continuously when crossing $V_{c}$, whereas it shows a discontinuity in the case of a first order phase transition.

We find that now it is indeed important to use a staggered field, Eq. (18), as a variational SFA parameter. In Fig.5 results are shown with such an optimization (solid lines) and without (dashed lines). Whereas at $V=1.6$ both calculations show only the SDW minimum at $\delta=0$,

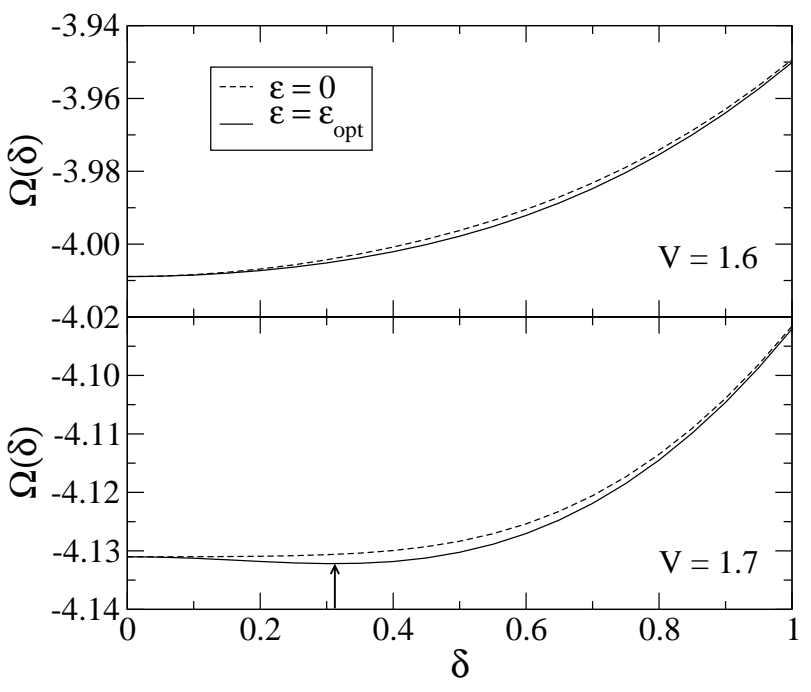

FIG. 5: Grand potential $\Omega$ as function of the mean-field parameter $\delta$ at $U=3$ calculated on a cluster with $N_{c}=8$ sites as reference system. Upper panel: $V=1.6$. Lower panel: $V=1.7$. Solid lines: With optimization of a staggered field. Dashed lines: Without optimization of a staggered field. The arrow marks the CDW minimum at $V=1.7$.

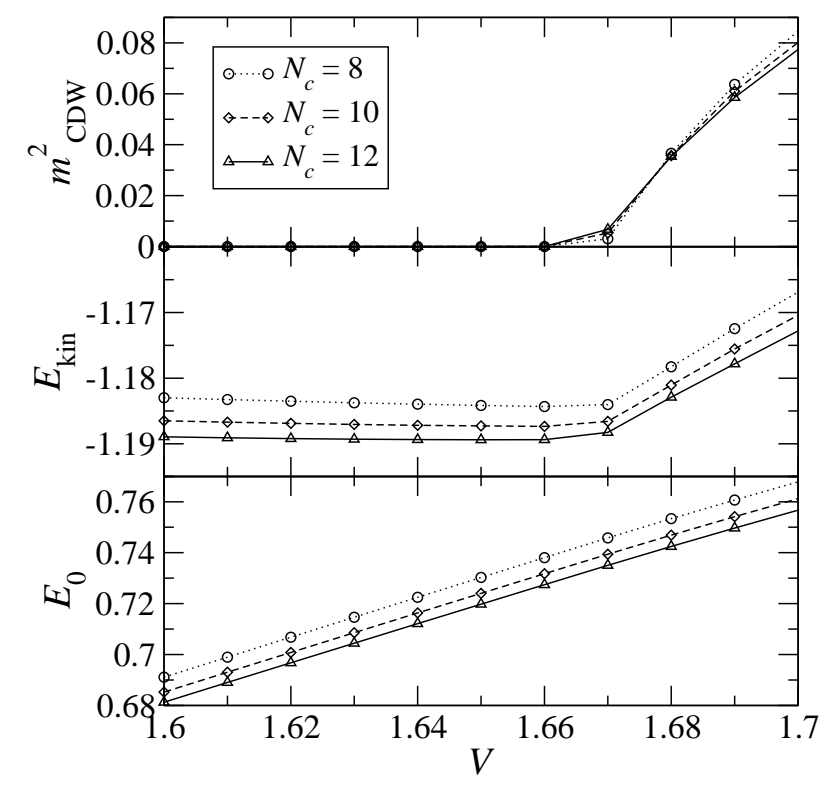

FIG. 6: Ground state energy $E_{0}$, kinetic energy $E_{\text {kin }}$, and order parameter $m_{\mathrm{CDW}}^{2}$ of the one-dimensional EHM at $U=3$ for cluster sizes $N_{c}=8$ (dotted), $N_{c}=10$ (dashed), and $N_{c}=12$ (solid line).

they differ at $V=1.7$ where the system should already be in the charge-ordered phase ${ }^{21.23 .24 .26}$ Without optimization of the staggered field, we would still find the SDW minimum at $\delta=0$, but with optimization the minimum shows up for a finite value of $\delta= \pm 0.31$ characteristic for the CDW phase.

For the determination of the critical value $V_{c}$, we calculated the ground state energy $E_{0}$, kinetic energy $E_{\text {kin }}$, 


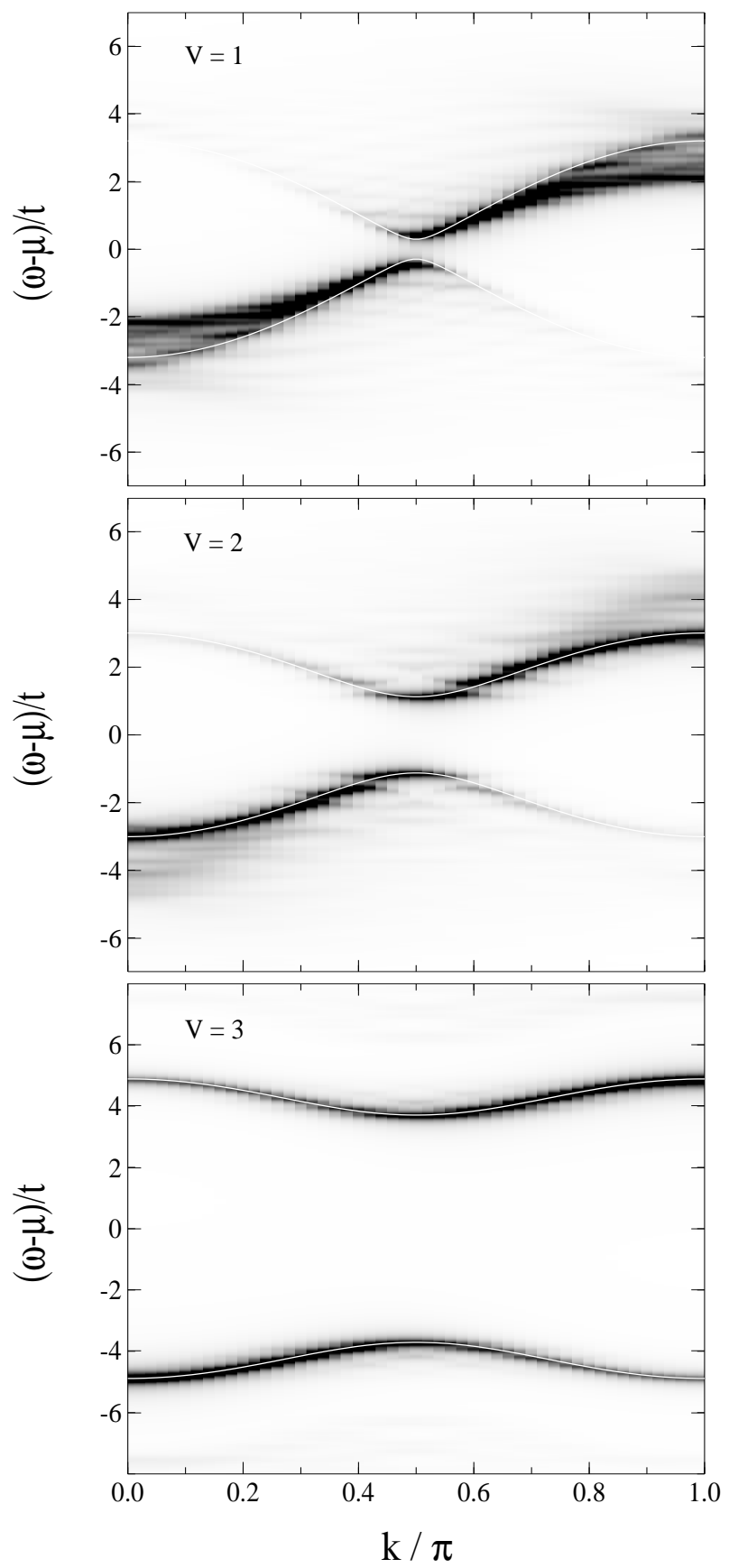

FIG. 7: Density plot of the spectral function $A(\mathbf{k}, \omega)$ of the one-dimensional EHM at $U=3$ calculated on a cluster of size $N_{c}=12$ with Lorentzian broadening $\sigma=0.1$. Darker regions represent larger spectral weight. From top to bottom: $V=1.0,2.0,3.0$. White lines are fits to a Hartree-Fock SDW/CDW dispersion (see text).

and the order parameter $m_{\mathrm{CDW}}$ at several values of $V$ shown in Fig.6] We performed no finite-size scaling like in Sec.IVA since we found that here the cluster sizes are too small for a systematic scaling. Different from Fig. [3 $E_{\text {kin }}, m_{\mathrm{CDW}}$, and the slope of the ground state energy are continuous across the transition point as required for a
TABLE II: Fitted values for the hopping matrix element $t_{\text {fit }}$, gap $\Delta_{\text {fit }}$, and $\Delta_{\text {HF }}$ within the Hartree-Fock approximation at $U=3$.

\begin{tabular}{|c|c|c|c|}
\hline & $t_{\text {fit }}$ & $\Delta_{\text {fit }}$ & $\Delta_{\mathrm{HF}}$ \\
\hline$V=0$ & 1.38 & 0.29 & 0.93 \\
$V=1$ & 1.59 & 0.29 & 0.93 \\
$V=2$ & 1.40 & 1.13 & 2.12 \\
$V=3$ & 1.59 & 3.71 & 4.28 \\
\hline
\end{tabular}

second order transition. From the kinetic energy and the order parameter calculated on a cluster of size $N_{c}=12$, we extract a critical value of $V_{c}=1.665(5)$, which is in good agreement with the critical value $V_{c} \approx 1.65$ obtained by QMC $\stackrel{21}{1}$ and diagonalization methods,$\stackrel{23.24}{=}$ and with $V_{c}=1.64(1)$ from DMRG calculations ${ }^{26}$ The slight difference is likely due to remaining finite-size effects. Moreover we made use of a single variational parameter only, namely the staggered field Eq. (18), and it can be expected that including more single-particle parameters in the SFA optimization procedure would give even more accurate results.

We would like to point out that in the present case of a second order phase transition, the most accurate way of calculating the mean-field parameter $\delta$ is to find the minimum in the grand potential including SFA optimization of single-particle parameters. Calculations on a cluster of size $N_{c}=12$ showed that without optimization the critical value would be $V_{c}=1.685(5)$. Compared to $V_{c}=1.665(5)$ this is further away from the values obtained by other methods as given above. Calculations with $\delta$ obtained self-consistently on an isolated cluster are insufficient. In this case one would get $V_{c}=1.735(5)$ for the $N_{c}=12$ cluster. This means that for a second order phase transition $\delta$ should be determined by minimizing the grand potential, whereas for first order transitions the self consistent determination was sufficient.

The spectral function $A(\mathbf{k}, \omega)$ at $V=1.0,2.0$, and 3.0, which has not been calculated previously, is depicted in Fig.7 We found that the spectral function at $V=1.0$ shows only minor differences to the spectral function of the Hubbard model $(V=0)$. The white lines in Fig. 7 are fits to a Hartree-Fock SDW/CDW dispersion. The parameters $t_{\text {fit }}$ and $\Delta_{\text {fit }}$ can be read off from Tab.III In the SDW phase at $V=0$ and $V=1.0$, the gap $\Delta_{\text {fit }}$ is constant. Similar to the case $U=8$ the agreement between $\Delta_{\text {fit }}$ and $\Delta_{\mathrm{HF}}$ is better in the CDW phase than in the SDW phase. The hopping parameter $t_{\text {fit }}$ increases when approaching the phase transition from below, similar to Tab. [1] but the fitted values for $t_{\text {fit }}$ are considerably smaller than in the case $U=8$.

\section{TWO DIMENSIONS}

The two-dimensional Hubbard model is one of the most intensely discussed models for strongly-correlated 

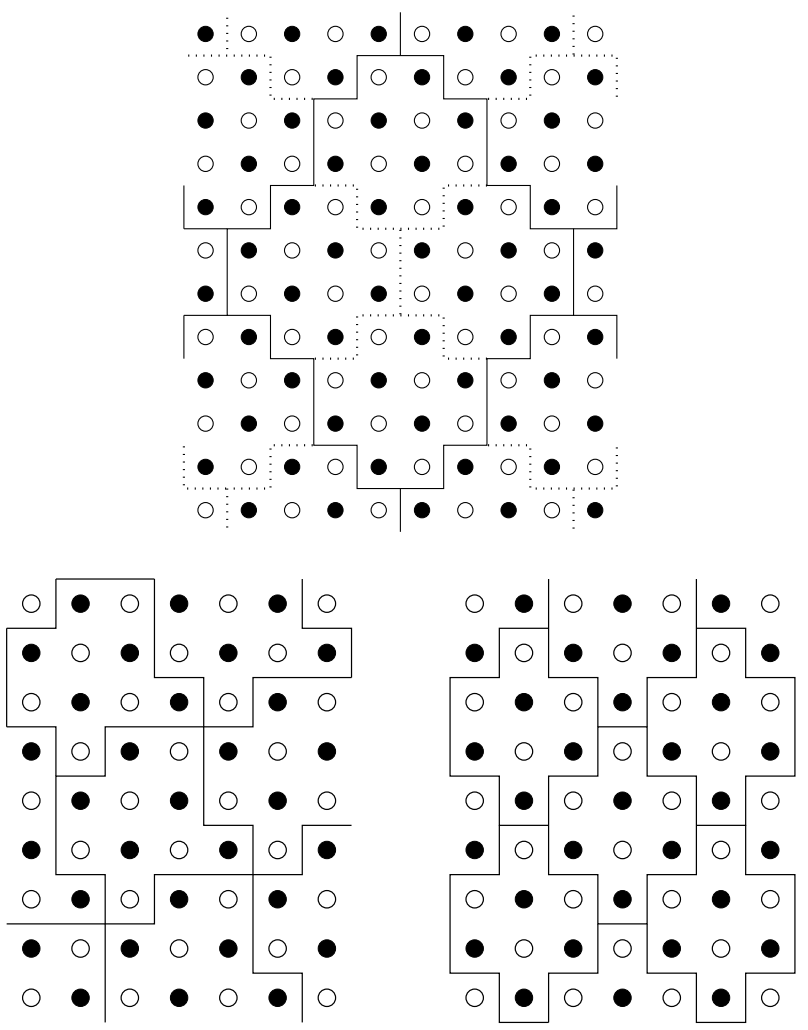

FIG. 8: Possible tilings of the two dimensional square lattice into clusters that allow for staggered ordering: $N_{c}=8$ (bottom right), $N_{c}=10$ (bottom left), super cluster with $N_{c}=48$ (top).

electron systems, especially in the context of hightemperature superconductivity. But different from the one-dimensional case, where many sophisticated methods have been used to investigate the extended Hubbard model as described in Sec.IV] only few studies have been done for the two-dimensional EHM. One reason for this is that many modern methods such as DMRG or fermionic loop-update QMC are difficult to apply to more than one spatial dimension. However, within our present approach, the extension to two dimensions is straightforward.

The two-dimensional EHM is defined by the Hamiltonian

$$
\begin{aligned}
H= & -t \sum_{\langle i j\rangle, \sigma}\left(c_{i, \sigma}^{\dagger} c_{j, \sigma}+\text { H.c. }\right)+U \sum_{i} n_{i \uparrow} n_{i \downarrow} \\
& +V \sum_{\langle i j\rangle} n_{i} n_{j}-\mu \sum_{i} n_{i},
\end{aligned}
$$

where $\langle i j\rangle$ connects nearest neighbors and the chemical potential is $\mu=U / 2+4 V$ at half filling. Early QMC studies ${ }^{29}$ showed that this model has a SDW-CDW transition similar to the one-dimensional case with transition point $V_{c} \approx U / 4$. But due to numerical difficulties it was impossible to determine the exact position and the order of the phase transition. Calculations within the Hartree-

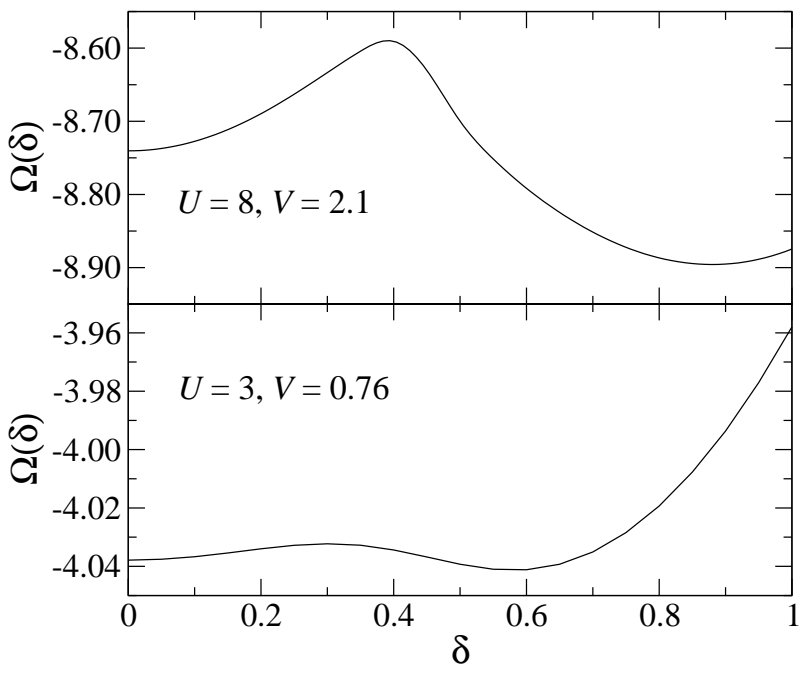

FIG. 9: Grand potential calculated on a cluster of size $N_{c}=8$ at $U=8, V=2.1$ (upper panel) and $U=3, V=0.76$ (lower panel).

Fock approximation ${ }^{30}$ showed two stable phases for the Hamiltonian Eq. (22) at half filling, the SDW and CDW phase, separated by a phase boundary at $V_{c}=U / 4$.

For the application of the method presented in Sec.II the two-dimensional square lattice has to be decoupled into clusters of finite size. Three possible tilings with different numbers of cluster sites $N_{c}$ are shown in Fig.8 Some care has to be taken concerning the staggered ordering. Whereas for clusters with $N_{c}=8$ and $N_{c}=10$ shown in Fig.8 the staggered ordering indicated by open and full circles is commensurate over the cluster boundaries, a straightforward decoupling into clusters of size $N_{c}=12$ is not possible. As one can easily see, a super cluster with $N_{c}=48$ consisting of four $N_{c}=12$ clusters has to be constructed in order to take into account the staggered ordering correctly. The Green's function of the super cluster can be calculated by switching off the hopping processes that connect the single $N_{c}=12$ clusters, in other words on bonds across the dotted lines in Fig.8. This gives a block-diagonal Hamiltonian which can be treated by the Lanczos algorithm. The switched off hopping processes are then incorporated again perturbatively, that means by including the corresponding hopping terms in the matrix $T_{a, b}^{\mathbf{R}, \mathbf{R}^{\prime}}$ in Eq. (4). Note that here the vectors $\mathbf{R}$ and $\mathbf{R}^{\prime}$ denote the super clusters and not the single $N_{c}=12$ clusters. Of course there are many other possible tilings like the $4 \times 3$ cluster used in Refs. 12 13 31, but also in that case a super cluster of $N_{c}=24$ has to be used.

We start the analysis of the two-dimensional EHM with the determination of the order of the phase transition. For this purpose we use the $N_{c}=8$ cluster shown in Fig. 8 and calculate the grand potential $\Omega(\delta)$ in the vicinity of the transition point at $U=8.0$ and $U=3.0$ as described in method(ii) in Sec.III Here we did not use a staggered field as variational parameter, because it does not 
change the qualitative shape of $\Omega(\delta)$ (see Figs. 2] and 5) and is therefore not necessary for the determination of the order of the transition. The result of this calculation is shown in Fig.9. At both values of $U$ we found three minima, located at $\delta=0$ and $\delta= \pm \delta_{\mathrm{CDW}}$. This indicates a first order phase transition, different from the one-dimensional EHM, where at $U=3.0$ the transition is of second order. We checked that this different behavior is not likely to be a finite size effect due to the small linear dimension of the two-dimensional $N_{c}=8$ cluster by calculating $\Omega(\delta)$ for the one-dimensional model with $N_{c}=4$ which still shows clear evidence of a second-order phase transition at $U=3$.

The fact that the system shows first order transitions at both $U=8.0$ and $U=3.0$ simplifies the subsequent calculations. As discussed in the previous section, one gets good results in the case of a first order transition by using a mean-field parameter $\delta$ determined self consistently on an isolated cluster, as described in method (i) in Sec.III] This procedure is much faster than the calculation of the grand potential for many values of $\delta$, which makes it possible to use the $N_{c}=48$ super cluster shown in Fig.8 We want to mention at this point that the calculation of the grand potential for the two-dimensional system is much more time consuming than for one dimension because of the larger number of $\mathbf{Q}$ points required in Eq. (8). For one dimension $L \approx 40$ is sufficient for convergence, whereas $L \approx 500$ is necessary for two dimensions. Nevertheless it is of crucial importance to use a cluster as large as possible, because the ratio of bonds treated exactly to mean-field decoupled bonds increases with increasing cluster size, especially pronounced for the two-dimensional square lattice. After having determined the mean-field parameter $\delta$ for the CDW phase self consistently, we also performed an SFA optimization of a staggered field, Eq. (18).

A few more words have to be said about calculations in the SDW phase $(\delta=0)$. Recent studies 20 of the pure Hubbard model revealed that it is important to take into account the long-range magnetic order for the accurate description of salient features of the system. This can be achieved by using a staggered magnetic field as variational parameter, given by

$$
\Delta_{a, b}=h \delta_{a, b} z_{\sigma} e^{i \mathbf{Q R}} \mathbf{R}_{a}
$$

with $z_{\sigma}= \pm 1$ for spin projection $\sigma=\uparrow, \downarrow$, and $h$ the strength of the field. Additionally it was argued that due to the connection of the hopping parameter $t$ and the magnetic exchange constant $J$, results could be further improved by letting the hopping in the clusters be of strength $t^{\prime}$ and optimizing the staggered magnetic field and $t^{\prime}$ simultaneously. Therefore we use

$$
\Delta_{a, b}=h \delta_{a, b} z_{\sigma} e^{i \mathbf{Q R} \mathbf{R}_{a}}-\tau \delta_{\langle a b\rangle^{\prime}}
$$

where the symbol $\delta_{\langle a b\rangle}$ is equal to one for nearestneighbor bonds inside the cluster and zero otherwise. The field strength $h$ and $\tau=t^{\prime}-t$ are the variational parameters in the optimization procedure.

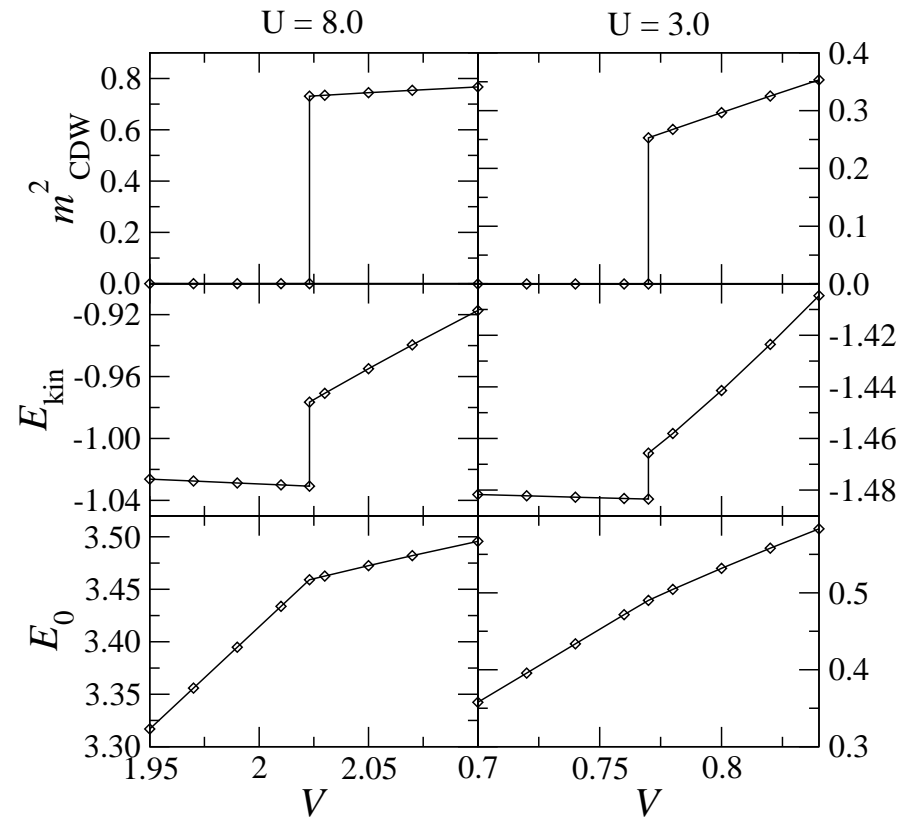

FIG. 10: Ground state energy $E_{0}$, kinetic energy $E_{\text {kin }}$, and order parameter $m_{\mathrm{CDW}}^{2}$ of the $2 \mathrm{D}$ EHM at $U=8.0$ (left) and $U=3.0$ (right). Calculations were done on a $N_{c}=48$ super cluster.

To sum up, the following steps are performed in the analysis using the $N_{c}=48$ super cluster: (i) First we determine the mean-field parameter $\delta_{\mathrm{CDW}}$ in the CDW phase self-consistently on an isolated cluster and (ii) use a staggered field Eq. (18) for an SFA optimization procedure. (iii) In the SDW phase $(\delta=0)$ the staggered magnetic field Eq. (23) and the intra-cluster hopping $t^{\prime}$ are optimized simultaneously. (iv) After determination of the SFA variational parameters we calculate the quantities we are interested in.

The results for the ground state energy, kinetic energy, and order parameter are shown in Fig. 10] At both $U=8.0$ and $U=3.0$, the behavior of a first order transition can be seen, where the change in the slope of $E_{0}$ is much stronger at $U=8.0$ than at $U=3.0$. This change at $U=8.0$ is even more pronounced than for the one-dimensional model at $U=8.0$. From Fig.10 we can extract the critical value $V_{c}$ of the phase transition by fitting $E_{0}$ to a straight line in the vicinity of the transition point, and for the $N_{c}=48$ super cluster we find $V_{c}=2.023(1)$ at $U=8.0$, and $V_{c}=0.770(3)$ at $U=3.0$. These values of $V_{c}$ are much closer to the Hartree-Fock result $V_{c}=U / 4$ than for one dimension. Within our approach we cannot clarify whether this is an intrinsic feature of the two-dimensional model or it is an artifact of the approximation due to the larger number of meanfield decoupled bonds.

The SFA variational parameters in the SDW phase near the phase transition point are found to be almost independent of the interaction $V$. At $U=8$, the optimization resulted in $t^{\prime} \approx 1.1$ for the intra-cluster hopping and 
$h \approx 0.14$ for the staggered magnetic field. The optimization of just one single parameter leads to $\left.t^{\prime} \approx 1.03\right|_{h=0}$ and $\left.h \approx 0.12\right|_{t^{\prime}=t}$, and the value of $\Omega$ also differs significantly from the value obtained by the simultaneous optimization of $t^{\prime}$ and $h$. This means that due to the strong connection between the magnetic ordering and the hopping matrix element it is important to optimize $t^{\prime}$ and $h$ simultaneously in order to get the best approximation for the physics in the thermodynamic limit. In the charge ordered phase the dependence of the variational parameter, Eq. (18), on the interaction $V$ is larger with $\varepsilon=0.08$ at $V=2.01$ and $\varepsilon=0.22$ at $V=2.1$. A similar behavior can be found at $U=3$ : In the SDW phase the variational parameters $t^{\prime} \approx 1.61$ and $h \approx 0.15$ are almost independent of $V$. In the CDW phase we get $\varepsilon=-0.03$ at $V=0.76$ and $\varepsilon=-0.18$ at $V=0.84$.

Whereas the application of the magnetic staggered field exhibits the symmetry $h \rightarrow-h$, this is not the case for the staggered field Eq. (18), because the symmetry is already broken by the mean-field decoupling. We found no stationary point of $\Omega$ for finite $h$ in the CDW phases.

The spectral function at $U=8$ in the SDW phase $(V=1.0)$ and in the CDW phase $(V=3.0)$ is shown in Fig.11] We found that the spectral function at $V=1.0$ is very similar to the spectral function of the Hubbard model $(V=0){ }^{20}$ One can see that the spectrum mainly consists of four features, two high-energy Hubbard bands and two low-energy quasi-particle bands, separated by a gap in the spectrum. The dispersion of these low-energy excitations in the SDW phase differs significantly from the Hartree-Fock shape shown as white lines in the upper panel of Fig.11] which does not account for the splitting into coherent low-energy bands and high-energy Hubbard bands. The fit parameters were $t_{\text {fit }}=1.34$ and $\Delta_{\text {fit }}=$ 2.51. The width of the coherent bands $|\omega(X)-\omega(\Gamma)| \approx$ 1.25 is rather set by the magnetic exchange $J$, consistent with QMC calculations at $V=0.32 .33$ The black lines are fits to $E_{\mathbf{k}}= \pm\left[-\Delta+J / 2\left(\cos k_{x}+\cos k_{y}\right)^{2}\right]$ which accounts better for the dispersion of the low-energy bands than the Hartree-Fock dispersion ${ }^{32}$ The fit parameters were $\Delta_{\text {fit }}=2.69$ and $J_{\text {fit }}=-0.63$, which is in good agreement with the second-order perturbation theory result $J=-4 t^{2} /(U-V)=-0.57$. In the CDW phase the white lines correspond to Hartree-Fock dispersions with fit parameters $\Delta_{\text {fit }}=7.69$ and $t_{\text {fit }}=1.16$, and different from the SDW phase they agree well with the excitations of $A(\mathbf{k}, \omega)$.

Fig.12 displays the spectral function at $U=3$ at interactions $V=0.5$ and $V=1.0$, respectively. The white lines again correspond to Hartree-Fock dispersions with fit parameters $t_{\text {fit }}=1.06, \Delta_{\text {fit }}=0.64$ at $V=0.5$, and $t_{\text {fit }}=1.07, \Delta_{\text {fit }}=1.82$ at $V=1.0$, respectively. As in the case $U=8$ the dispersion of the coherent low-energy bands in the SDW phase differs from the Hartree-Fock prediction, but in this case the deviation is much smaller. We did not find an accurate functional form in order to fit the low-energy excitations, but nevertheless we can extract the value of $J$ from the band with of the coherent

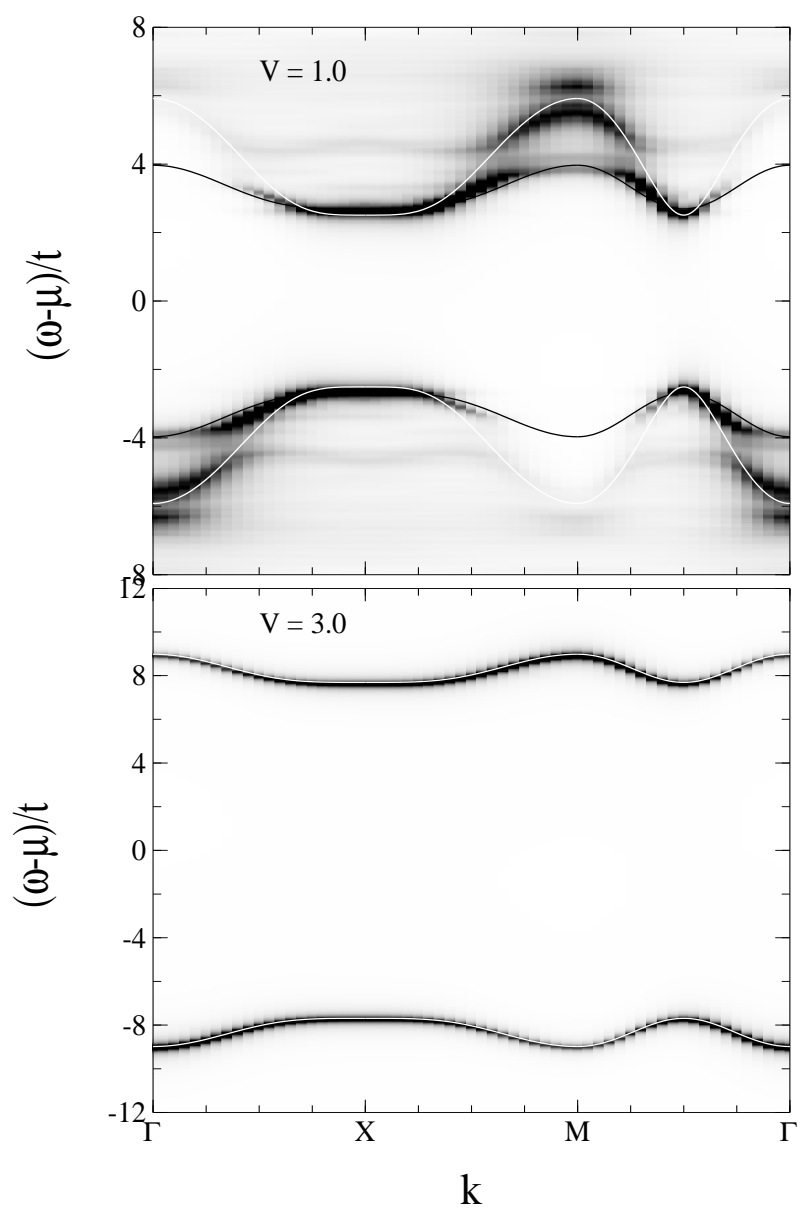

FIG. 11: Density plot of the spectral function $A(\mathbf{k}, \omega)$ of the two-dimensional EHM at $U=8$ calculated on a $N_{c}=48$ super cluster with broadening $\sigma=0.1$. Darker regions represent larger spectral weight. Top: $V=1.0$. Bottom: $V=3.0$. White lines are fits to Hartree-Fock dispersions. For the meaning of the black lines at $V=1.0$ see text.

bands yielding $J=-(1 / 2)|\omega(X)-\omega(\Gamma)| \approx-1.57$. This value is again in good agreement with the perturbation theory result $J=-4 t^{2} /(U-V)=-1.6$.

We would like to mention that our results at $U=3$ in the SDW phase are qualitatively different from QMC results at $U=3, V=0$, and inverse temperature $\beta=3 t, 34$ where the spectral function shows metallic behavior with no gap around the Fermi energy. This difference may be due to temperature effects or due to poor resolution of the Maximum-Entropy inversion of QMC correlation functions.

At both $U=8$ and $U=3$, one can easily see that agreement of the Hartree-Fock dispersions with the lowenergy excitations of $A(\mathbf{k}, \omega)$ is better in the CDW phase than in the SDW phase. In addition the gap $\Delta_{\mathrm{HF}}$ calculated within the Hartree-Fock approximation is much closer to the fitted gap $\Delta_{\text {fit }}$ in the CDW phase (e.g. $\Delta_{\mathrm{HF}}=7.76, \Delta_{\mathrm{fit}}=7.69$ at $\left.U=8, V=3\right)$ than in the SDW phase (e.g. $\Delta_{\mathrm{HF}}=3.57, \Delta_{\text {fit }}=2.51$ at $U=8$, $V=0$ ). Therefore we conclude that in the CDW phase 


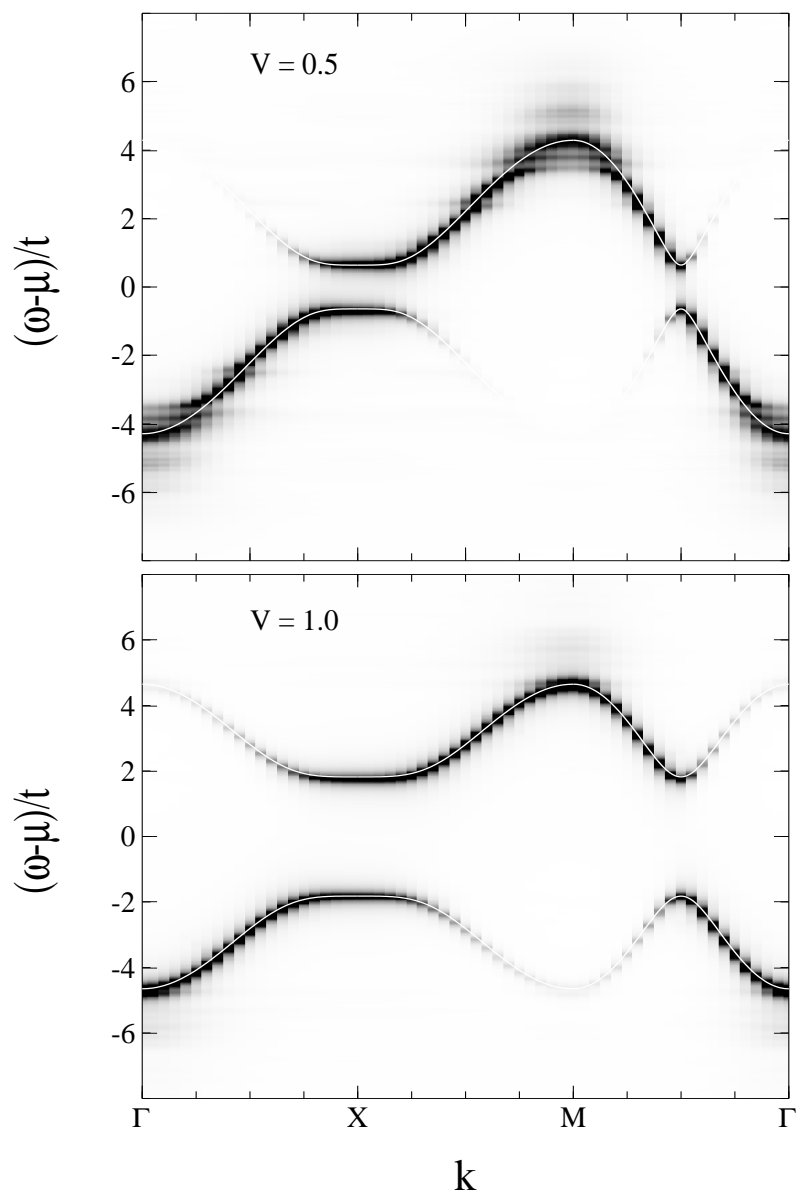

FIG. 12: Same as Fig.11] but at $U=3$. Top: $V=0.5$. Bottom: $V=1.0$.

charge fluctuations play only a minor role compared to the SDW phase, similar to the one-dimensional system.

\section{CONCLUSIONS}

In this paper we have presented a generalization of the variational cluster perturbation theory to extended Hubbard models at half filling. The method is based on the self-energy-functional approach (SFA) which uses dynamical information of an exactly solvable system (reference system $H^{\prime}$ ) in order to approximate the physics in the thermodynamic limit. For the application of this method, a mean-field decoupling of the inter-cluster part of the nearest-neighbor Coulomb interaction is performed first. After this step, one is left with a Hamiltonian which couples the different clusters via the hopping only and which can be treated by the known (variational) CPT procedure. The mean-field decoupling yields effective onsite potentials on the cluster boundaries as external parameters of the Hamiltonian. These parameters are determined either self-consistently on an isolated cluster (sufficient for the study of first order phase transitions) or by determination of the minimum of the SFA grand potential.

In order to test the accuracy of our approach we applied the method to the extended Hubbard model in one dimension, because results from other methods like QMC and DMRG are available for comparison. At $U=8$ the results for the critical interaction $V_{c}$, the groundstate energy, kinetic energy, and charge order parameter showed excellent quantitative agreement with previous QMC studies. At $U=3$ our method predicted a second-order phase transition with transition point $V_{c}=1.665(5)$ again in good agreement with previous studies. The ground state energy, kinetic energy, and order parameter do not seem to have been calculated before.

In addition we calculated the spectral function for several values of the interaction $V$, which has not been done previously. At both $U=8$ and $U=3$, we found evidence for spin-charge separation in the SDW phase, but not in the CDW phase. By fitting the bands by HartreeFock dispersions we found that the hopping parameter is strongly renormalized. The agreement between the fitted value of the gap and the value within the HartreeFock approximation was much better in the CDW phase than in the SDW phase giving rise to the conclusion that charge fluctuations play a minor role in the CDW phase.

Whereas the application of sophisticated methods like DMRG or fermionic loop-update QMC to more than one dimension is difficult, this extension is straightforward within the present approach. We were thus able to perform the first non-perturbative study of the twodimensional extended Hubbard model on a square lattice at half filling and zero temperature beyond HartreeFock. We found first order transitions at both $U=8$ and $U=3$ with transition points $V_{c}=2.023(1)$ and $V_{c}=0.770(3)$ for an $N_{c}=48$ super cluster, respectively. The spectral function in the SDW phase shows coherent low-energy quasi-particle excitations with band width set by the magnetic exchange constant $J$, and an incoherent background, consistent with previous QMC studies for the Hubbard model at $V=0$. The Hartree-Fock prediction differs significantly from the low-energy feature and does not describe the splitting into coherent quasiparticle bands and incoherent background. In the CDW phase the Hartree-Fock dispersions account much better for the excitations, and no additional low-energy features caused by a magnetic origin could be found. Similar to one dimension the agreement between the Hartree-Fock approximation and the low-energy excitations obtained by the present method is much better in the CDW phase, confirming that charge fluctuations are less important in the charge-ordered phase than in the SDW phase.

In this paper we applied our method to half-filled systems only, but one can study ordering phenomena at other fillings, too, as long as the possible order patterns are commensurate with the shape of clusters used as reference system. With some effort it is also possible to study phases with long wave-length charge density waves by coupling several clusters to a super cluster and ap- 
plying appropriate continuity conditions between the individual clusters within the super cluster. In addition the application to systems with lattice geometry different from the two-dimensional square lattice, e.g. ladder materials, is an interesting subject for further studies. Work in this direction is in progress.

\section{Acknowledgments}

This work has been supported by the Austrian Science Fund (FWF), projects P15834 and P15520. M. A. is supported by a doctoral scholarship program of the Austrian Academy of Sciences. We gratefully acknowledge useful and stimulating discussions with M. Hohenadler, W. Koller, E. Arrigoni, and C. Dahnken.

\section{APPENDIX A: MEAN-FIELD SOLUTION AND FREE ENERGY}

In this section we show that a mean-field solution obtained self consistently is directly connected to a minimum in the free energy. For simplicity let us assume that we have only two different mean-field parameters $\lambda_{A}=1-\delta$ and $\lambda_{B}=1+\delta$, see also Sec.III We can write the mean-field decoupled Hamiltonian Eq. (16) as

$$
H_{\mathrm{MF}}(\delta)=H_{\mathrm{MF}}^{(0)}+\sum_{\mathbf{R}} H_{\mathrm{MF}}^{(1)}(\mathbf{R}, \delta),
$$

where $H_{\mathrm{MF}}^{(0)}$ includes all terms independent of the meanfield parameters. According to the third line in Eq. (13), $H_{\mathrm{MF}}^{(1)}(\mathbf{R}, \delta)$ is given by

$$
\begin{aligned}
& H_{\mathrm{MF}}^{(1)}(\mathbf{R}, \delta)=V \sum_{[i j]}\left[n_{\mathbf{R} i} \lambda_{B}+n_{\mathbf{R} j} \lambda_{A}-\lambda_{A} \lambda_{B}\right] \\
& =V \sum_{[i j]}\left[n_{\mathbf{R} i}(1+\delta)+n_{\mathbf{R} j}(1-\delta)-\left(1-\delta^{2}\right)\right]
\end{aligned}
$$

where we assumed without loss of generality that the bonds $[i j]$ connect sites $i$ on sublattice $A$ with sites $j$ on sublattice $B$. The free energy of the system is given by

$$
\begin{aligned}
F & =-\frac{1}{\beta} \ln Z=-\frac{1}{\beta} \ln \operatorname{tr} e^{-\beta H_{\mathrm{MF}}(\delta)} \\
& =-\frac{1}{\beta} \ln \operatorname{tr} \exp \left[-\beta H_{\mathrm{MF}}^{(0)}-\beta \sum_{\mathbf{R}} H_{\mathrm{MF}}^{(1)}(\mathbf{R}, \delta)\right]
\end{aligned}
$$

Taking the derivative with respect to $\delta$ yields

$$
\frac{\partial F}{\partial \delta}=V \sum_{\mathbf{R}}\left\langle\sum_{[i j]}\left[n_{\mathbf{R} i}-n_{\mathbf{R} j}+2 \delta\right]\right\rangle .
$$

All clusters are equivalent, therefore we suppress the in$\operatorname{dex} \mathbf{R}$ in the following. Setting this derivative to zero we get the self-consistency condition

$$
\sum_{[i j]}\left[\left\langle n_{i}\right\rangle-\left\langle n_{j}\right\rangle+2 \delta\right]=0
$$

For one dimension, Eq. A5 is given by

$$
\left\langle n_{N}\right\rangle-\left\langle n_{1}\right\rangle=2 \delta
$$

because in this case we have only one decoupled bond $[1 N]$ with site $1(\mathrm{~N})$ belonging to sublattice $A(B)$, respectively. To conclude, one can state that if self consistency, Eq. (A5), is fulfilled, then the free energy has an extremum with respect to the mean-field parameter $\delta$. By thermodynamic stability arguments this extremum always has to be a minimum.
1 W. Metzner, Phys. Rev. B 43, 8549 (1991).

2 S. Pairault, D. Sénéchal, and A.-M. S. Tremblay, Phys. Rev. Lett. 80, 5389 (1998).

3 S. Pairault, D. Sénéchal, and A.-M. S. Tremblay, Eur. Phys. J. B 16, 85 (2000).

4 E. Dagotto, Rev. Mod. Phys. 66763 (1994).

5 S. R. White, Phys. Rev. Lett. 69, 2863 (1992); Phys. Rev. B 48, 10345 (1993).

${ }^{6}$ For a review see A. Georges, G. Kotliar, W. Krauth, and M. J. Rozenberg, Rev. Mod. Phys. 68, 13 (1996).

7 M. Hettler, A. N. Tahvildar-Zadeh, M. Jarrell, T. Pruschke, and H. R. Krishnamurthy, Phys. Rev. B 58, 7475 (1998).

8 G. Kotliar, S. Y. Savrasov, G. Palsson, and G. Biroli, Phys. Rev. Lett 87, 186401 (2001).
${ }^{9}$ G. Biroli and G. Kotliar, Phys. Rev. B 65, 155112 (2002).

10 C. J. Bolech, S. S. Kancharla, and G. Kotliar, Phys. Rev. B 67, 075110 (2003).

11 C. Gros and R. Valenti, Phys. Rev. B 48, 418 (1993).

12 D. Sénéchal, D. Pérez, and M. Pioro-Ladrière, Phys. Rev. Lett. 84, 522 (2000).

13 D. Sénéchal, D. Pérez, and D. Plouffe, Phys. Rev. B 66, 075129 (2002).

14 M. G. Zacher, R. Eder, E. Arrigoni, and W. Hanke, Phys. Rev. Lett. 85, 2585 (2000).

15 M. G. Zacher, R. Eder, E. Arrigoni, and W. Hanke, Phys. Rev. B 65, 045109 (2002).

16 C. Dahnken, E. Arrigoni, and W. Hanke, J. of Low Temp. Phys. 126, 949 (2002).

17 M. Aichhorn, M. Daghofer, H. G. Evertz, and 
W. von der Linden, Phys. Rev. B 67, 161103R (2003).

18 M. Potthoff, Eur. Phys. J. B 32, 429 (2003).

19 M. Potthoff, M. Aichhorn, and C. Dahnken, Phys. Rev. Lett. 91, 206402 (2003).

20 C. Dahnken, M. Aichhorn, W. Hanke, E. Arrigoni, and M. Potthoff, cond-mat/0309407

21 P. Sengupta, A. W. Sandvik, and D. K. Campbell, Phys. Rev. B 65, 155113 (2002).

22 J. E. Hirsch, Phys. Rev. Lett. 53, 2327 (1984).

23 M. Nakamura, J. Phys. Soc. Jpn. 68, 3123 (1999).

24 M. Nakamura, Phys. Rev. B 61, 16377 (2000).

25 M. Tsuchiizu and A. Furusaki, Phys. Rev. Lett. 88, 056402 (2002).
26 E. Jeckelmann, Phys. Rev. Lett. 89, 236401 (2002).

27 M. Tsuchiizu and A. Furusaki, cond-mat/0308157

28 J. Voit, Phys. Rev. B 45, 4027 (1992).

29 Y. Zhang and J. Callaway, Phys. Rev. B 39, 9397 (1989).

30 B. Chattopadhyay and D. M. Gaitonde, Phys. Rev B 55, 15364 (1997).

31 D. Sénéchal and A.-M.S. Tremblay, cond-mat/0308625

32 R. Preuss, W. Hanke, and W. von der Linden, Phys. Rev. Lett. 75, 1344 (1995).

33 C. Gröber, R. Eder, and W. Hanke, Phys. Rev. B 62, 4336 (2000).

${ }^{34}$ C. Gröber, M. G. Zacher, and R. Eder, cond-mat/9810246 